\title{
La calidad de vida. Juicios de satisfacción y felicidad como indicadores actitudinales de bienestar
}

\author{
María Amérigo Cuervo-Arango \\ Universidad Complutense de Madrid
}

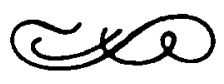

La preocupación por establecer una aceptable calidad en la vida de las personas desde una perspectiva social y política es una de las principales metas manifiestas a conseguir por los gobiernos occidentales. Estos han de procurar el bienestar de los individuos a su cargo. El estudio de la calidad de vida en los países desarrollados, ha tomado un auge considerable, sobre todo a partir de la segunda mitad de los años sesenta y, en concreto, desde una perspectiva psicológica, el estudio de esta temática se ha materializado en multitud de trabajos que intentan medir la calidad de vida de los ciudadanos -o de alguna faceta de la misma - a partir de lo que se conoce como indicadores sociales (Campbell, Converse y Rodgers, 1976; Andrews y Withey, 1976; serían ejemplos de estudios globales de calidad de vida). En dichos trabajos, interesa fundamentalmente el bienestar percibido, medido a través de juicios de satisfacción y/o felicidad.

Independientemente de las cuestiones metodológicas, la mayoría de los trabajos, tanto a nivel social, político o psicológico, se caracterizan por una ausencia de definiciones conceptuales con respecto a los términos que tratan. Calidad de vida, bienestar, felicidad o satisfacción son a menudo utilizados en un mismo sentido, de forma intercambiable, lo que dificulta en gran medida una aproximación teórica al estudio de la calidad de vida.

Por eso este artículo pretende detenerse a reflexionar acerca de estas cuestiones conceptuales con intención de exponer en alguna medida cuál es la situación actual a la hora de concebir tales términos claves.

\section{ALGUNAS CUESTIONES CONCEPTUALES}

Para empezar cabría distinguir entre el discurso «macronivel» en donde quedarían englobados políticos y sociólogos y el discurso «micronivel» característico del quehacer psicológico. En función de que se emplee un discurso u otro, los términos adquieren distinta significación que, en ocasiones, no siempre es recogida explícitamente. Tal es el caso del término bienestar.

Cuando se acude a la literatura anglosajona al repecto, se observan dos términos distintos para una misma traducción castellana del bienestar: «Well-being» 
y «welfare» y aunque en castellano sólo existe una traducción posible, parece que entre ambos se dan ciertas diferencias que han sido subrayadas por algunos estudiosos del bienestar.

Si se observa la literatura psicológica anglosajona sobre el bienestar, ésta hace referencia al término «well-being» mientras que «welfare» aparece más referido a cuestiones políticas o de gran alcance social; así no es extraño oír hablar en los discursos políticos del estado del bienestar - welfare state - o del bienestar general de la sociedad - general welfare - En este sentido iría la definición de Moix (1986) cuando considera el bienestar (welfare) de un individuo dotado de tres características: 1) Objetividad: «No es una cuestión de estados subjetivos de conciencia, sino de condiciones y circunstancias objetivas» (p. 23); 2) externalidad, en el sentido de que es directamente observable desde el exterior y 3) minimalidad o que requiere de ciertos requisitos mínimos que han de verse satisfechos si se quiere lograr ese estado de bienestar.

Es aquí, en el momento en que se habla de bienestar como welfare, cuando surge la identificación en algunos contextos con el término calidad de vida. Se habla tanto del estado del bienestar o del bienestar de la sociedad como de la calidad de vida de los individuos. Cuando el bienestar se refiere a la sociedad como un ente, cuando es considerado como un valor, es decir, cuando es empleado en boca de los políticos, bienestar y calidad de vida se identifican.

A este respecto es interesante considerar las reflexiones que sobre el surgimiento del concepto calidad de vida quedan reflejadas en un trabajo publicado por la Dirección General del Medio Ambiente (1979) sobre la calidad de vida en España y llevado a cabo por el grupo de «EMOPUBLICA». Según se desprende del mismo, habría que considerar el término calidad de vida como el sustituto - desde una perspectiva política - del término de bienestar (welfare); y éste a su vez provendría de un intento de objetivar y de extender al campo de lo público y social el concepto de felicidad. Según los autores, «... en un movimiento que podría calificarse con propiedad como dialéctico, el paso del tema del bienestar al de calidad de vida supone una recuperación de la felicidad» (p. 21).

El hecho de intentar extender el concepto de felicidad al campo de lo social, de lo público, supone una socialización de la moral que, en palabras de Aranguren (1989), es precisamente lo que pretende la sociedad del bienestar. El bienestar, en este sendo utilitarista, aparece en boca de Díaz (1987, p. 155) cuando citando al británico J. Stuart Mill afirma que éste «nunca cuestionó que el Estado fuese, por promotor del Estado de Bienestar, artífice e impulsor privilegiado de felicidad». Cabría preguntarse entonces si proporciona el Estado del Bienestar felicidad a sus miembros. Quizá la disfuncionalidad del Estado haya convertido el término bienestar en un mero eufemismo, que ha de ser reemplazado por otro más moderno y cargado de un nuevo espíritu, como así parece serlo, en la actualidad, el de calidad de vida.

Volviendo de nuevo a la cuestión de los dos referentes anglosajones del término castellano bienestar, cabría decir según lo visto hasta ahora que és welfare el que se identifica con calidad de vida. Sin embargo el término well-being, más utilizado por la literatura psicológica, es, en la mayor parte de los casos, considerado como un componente de esa calidad de vida. Así, la definición que Levi y Andersson $(1980$, p. 6) ofrecen del término calidad de vida iría en este sentido: «Por este concepto entendemos una medida compuesta de bienestar físico, mental y social, tal y como lo percibe cada individuo y cada grupo, y de felici- 
dad, satisfacción y recompensa». La calidad de vida estaría compuesta, por tanto, por bienestar percibido (well-being), felicidad y satisfacción.

No hay muchas definiciones de calidad de vida desde una perspectiva psicológica. La psicología se ha hecho eco de esta cuestión, como exponen Jiménez Burillo et al. (1984), «... después de que este concepto pasara a formar parte del vocabulario político, y lo ha hecho como consecuencia de sus preocupaciones por la felicidad y el bienestar del individuo» (p. 88). El estudio del bienestar individual y grupal, por tanto, es clave a la hora de trabajar sobre el concepto de calidad de vida desde una perspectiva psicológica. Así parece matizarlo Blanco (1985, p. 175) cuando afirma que hay pocas definiciones del concepto calidad de vida, pero que «es posible encontrar alusiones directas al tema, no al concepto en sí, en aquellos estudios que se han aproximado desde una u otra perspectiva al fenómeno del bienestar humano». Los estudios psicológicos sobre la calidad de vida, como se comentó en un principio, aluden por lo general al bienestar como componente de esa calidad de vida, como un indicador subjetivo, medido a través de juicios de satisfacción y felicidad.

Hasta ahora lo que se acaba de exponer quedaría resumido de forma sinóptica en la Figura I:

FIGURA I

Relación de conceptos relativos a la calidad de vida.

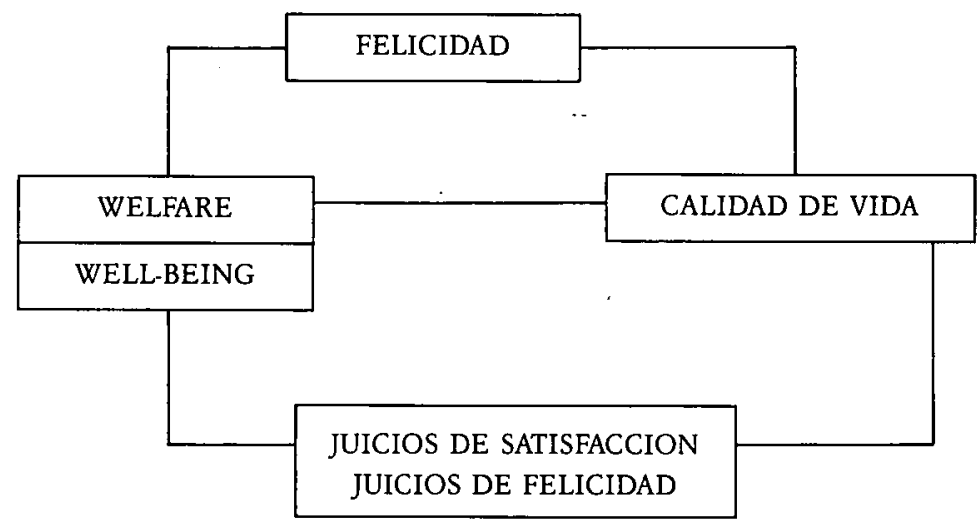

Según esta figura, bienestar, considerado éste en el sentido de welfare, y calidad de vida serían en realidad una misma cuestión. Ambos hacen referencia a la felicidad y de hecho deben su existencia a ella, considerada, como fin último hacia el que tiende el ser humano. El objetivo precisamente del welfare o de la calidad de vida es socializar la felicidad, que todos los individuos puedan alcanzarla. Desde una perspectiva psicológica y, por tanto, descendiendo del nivel macrosocial al nivel individual o grupal, el estudio de la calidad de vida se convierte en el estudio subjetivo del bienestar individual o grupal, del well-being. ¿A qué se debe el bienestar de un individuo o grupo? Componente, por tanto, esencial del concepto calidad de vida. Los juicios sobre la satisfacción y/o felicidad que un individuo o grupo experimenta con su vida o con cualquier faceta de la misma, son utilizados para determinar el grado de bienestar percibido, 
de well-being y, por tanto, pueden considerarse como indicadores subjetivos de la calidad de vida.

\section{MEDICION DE LA CALIDAD DE VIDA}

Como se comentó anteriormente, la gran mayoría de estudios sobre esta temática en los países desarrollados, se materializan en multitud de trabajos que intentan medir la calidad de vida de los ciudadanos a partir de los denominados indicadores sociales. Dichos trabajos, durante aproximadamente una década y principalmente desde mediados de los sesenta, tienen gran apogeo y se recogen en revistas especializadas tales como, entre otras, la Social Indicators $R e$ search a partir de 1973 .

Uno de los problemas fundamentales que motivó el surgimiento de los primeros estudios sobre esta temática era que los tradicionales informes emitidos por los gobiernos sobre la calidad de vida no eran indicativos de tal, pues únicamente se basaban en criterios de carácter económico y cuantitativo, meramente descriptivos de lo que ellos denominaban estado de la calidad de vida de un país. Era necesario incorporar elementos subjetivos, psicológicos, sobre cómo las personas percibían y valoraban sus propias condiciones en el trabajo, la salud, la vivienda, o en la vida en general. Pena (1977, p. 13) recoge algunas de las reflexiones del grupo de trabajo de la Organización de Cooperación y Desarrollo Económico (O.C.D.E.) sobre indicadores sociales en 1973: «La forma en que los individuos y los grupos perciben los aspectos fundamentales de su bienestar es un elemento necesario e importante del programa de los indicadores sociales».

Así los indicadores sociales surgieron como complemento indispensable para la medición de la calidad de vida.

No obstante, el tema del establecimiento de un sistema de indicadores ha sido criticado por diversos tipos de cuestiones, las cuales resultarían demasiado extensas para recoger aquí1. Uno de los principales problemas con los que se enfrentó esta temática era el de la objetividad versus subjetividad de los indicadores sociales. De esta forma, se planteó una fuerte polémica entre los partidarios de indicadores subjetivos y entre aquellos que opinaban que lo único medible y válido eran criterios objetivos, tales como tasas de crimen, nivel de ruido, grado de desempleo, etc. La crítica fundamental al empleo de indicadores subjetivos provenía de la dificultad de su medición.

Es ciertamente difícil medir sentimientos, percepciones, actitudes, etc. No obstante, la necesidad de incluir estos criterios subjetivos en los indicadores de calidad de vida es patente: desde la crítica postmarxista a ese ideal de hombre como homo economicus, el hombre materialista, donde la posesión material implica la felicidad; pasando por la teoría humanista de Abraham H. Maslow.

Levi y Andersson (1980) ofrecen una exposición muy clara al hecho de que no exista necesariamente una concordancia entre incremento de los recursos económicos e incremento de la satisfacción individual: «(...) La razón fundamental de esta falta de concordancia, aparentemente paradójica, es que por encima de cierto nivel mínimo de vida, el determinante fundamental de la calidad de la vida individual es el "ajuste" o la "coincidencia" entre las características de la situación (de exigencias y oportunidades) y las expectativas, capacidades y necesidades del individuo tal y como las percibe él mismo» (p. 59). Estos autores 
afirman que al aumentar el nivel material también aumentan las esperanzas, luego la satisfacción no aumentaría. No obstante, téngase en cuenta que las esperanzas son siempre manipulables, y esto, desde el punto de vista del que tiene en su mano la toma de decisión política, es de todo punto interesante. Quizá así expuesto, el hecho de que la esperanza pueda manipularse puede resultar un tanto escalofriante, aunque no menos cierto.

En la misma línea cabría citar los comentarios que Walker y Rosser (1988) realizan sobre el término calidad de vida, al afirmar que dicho término «representa un amplio espectro de dimensiones de la experiencia humana que van desde aquellas asociadas a las necesidades vitales, tales como comida y cobijo, a aquellas asociadas con desarrollar un sentido de plenitud y felicidad personal» (p. 11). Hay por tanto otras cuestiones de carácter subjetivo que están íntimamente unidas a la calidad de vida de las personas. El bienestar percibido es uno de tales aspectos y el hecho de que exista una dificultad a la hora de medirlo no justifica la necesidad de obviarlo.

Como una forma de determinar el grado de bienestar percibido, han venido considerándose tradicionalmente los juicios de satisfacción y felicidad que el individuo experimenta con su vida o con determinadas facetas de la misma. Se trata de estudiar, en definitiva, la actitud del sujeto hacia su vida en general, o hacia ciertos aspectos tales como la salud, el trabajo, la vivienda, el ocio, etc. Partiendo de la clásica visión tripartita de Rosenberg y Hovland sobre el concepto de actitud, dos componentes de la misma, el cognitivo y el afectivo, están implicados profundamente en los juicios evaluativos sobre el bienestar individual.

A partir de este momento, se tratará de poner de manifiesto algunas cuestiones sobre estos componentes relativos a las formas más ampliamente utilizadas de medir el bienestar individual percibido.

\subsection{Bienestar percibido: juicios de satisfacción y felicidad}

Como acaba de comentarse, cuando un sujeto emite un juicio de felicidad o satisfacción, bien con su propia vida o bien con un dominio específico de la misma; de alguna forma está expresando verbalmente su actitud hacia su vida o hacia ese dominio vital. Una de las principales polémicas que se ha generado en torno a este tema es el de intentar determinar en qué medida la felicidad y la satisfacción son de carácter afectivo o cognitivo, intento que puede resultar un tanto baldío debido a la dificultad de separar los componentes afectivo y cognitivo de la actitud, como así lo han puesto de manifiesto numerosas investigaciones a este respecto (Ajzen, 1988).

No obstante, puede resultar interesante exponer tal polémica cara a reflexionar sobre los conceptos de satisfacción y felicidad ya que el afán de encontrar las causas de felicidad y satisfacción en los individuos, de obtener resultados palpables, ha llevado en muchos casos a estudiar más el instrumento que el objeto de estudio en sí mismo. Así, es frecuente encontrar en investigaciones sobre calidad de vida multitud de trabajos empíricos, pero pocos estudios donde realmente se reflexione acerca de ambos conceptos.

Antes de entrar a discutir en qué medida los juicios de satisfacción y felicidad poseen un carácter cognitivo o afectivo, sería interesante analizar comparativamente el significado de dichos conceptos, ya que éste es a menudo intercambiable. ¿Es lo mismo satisfacción que felicidad? ¿Se alude a una misma idea cuando se emplean ambos términos? 


\section{6}

Hay autores que así lo consideran. Por ejemplo, Cantril (1965), en su estudio transcultural sobre los temores y esperanzas relevantes en la vida de las personas, tiende a utilizar de forma idéntica los juicios de satisfacción y felicidad. Campbell, Converse y Rodgers (1976), por el contrario, establecen la objeción de que es posible, por ejemplo, observar que, en general, los jóvenes se consideran más felices que los ancianos, pero a menudo también manifiestan estar más insatisfechos, por lo que se trataría de conceptos distintos.

De hecho, estos autores hallaron la correlación entre las puntuaciones más altas obtenidas en juicios de satisfacción - medidos a través de una escala de siete puntos, desde completamente satisfecho hasta completamente insatisfechoy las puntuaciones de los sujetos que habían contestado «muy feliz», en una escala de tres puntos - muy feliz, bastante feliz y no demasiado feliz-. El resultado fue una correlación de 0,50 ; lo que indica que empíricamente existe un solapamiento, pero no una total identificación entre ambos conceptos. No obstante, no puede afirmarse que las escalas para medir tales conceptos fueran válidas ${ }^{2}$.

Si se recurre a una fuente clásica como es el Diccionario de la Lengua Castellana, podrían encontrarse dos definiciones del término felicidad, dentro de la temática que nos ocupa: ésta sería considerada como «situación del ser para quien las circunstancias de su vida son tales como las desea. Estado de ánimo circunstancial del que consigue algo que contribuye a esa situación». Por otra parte el concepto satisfacción es definido como «estado de ánimo experimentado con lo que satisfaces ${ }^{3}$.

Realmente, la diferenciación entre ambos términos no parece sencilla. La segunda acepción del término felicidad y la definición de satisfacción son bastante coincidentes. Ambas aluden a estados de ánimo positivos en los que se encuentra el individuo en un momento concreto de su vida, es decir, son de carácter pasajero; un estado que se experimenta en un tiempo concreto, pero que no es estable, algo fundamentalmente afectivo. Sin embargo, entre la definición de satisfacción y la primera acepción del término felicidad se puede observar alguna diferencia. Así, esta última proviene de una comparación que el sujeto establece entre lo que posee en su vida y lo que desea de la misma; de tal forma que si posesión y deseo coinciden, el sujeto estará en una situación de felicidad. Esta acepción de felicidad parece aludir a algo de carácter más estable que las anteriores y el hecho de ser claramente comparativa lleva a pensar en mecanismos cognitivos. Por tanto, podría establecerse una primera aproximación en el sentido de otorgar un carácter más afectivo a la satisfacción y uno más cognitivo a la felicidad.

Hay otros autores como Argyle (1987) que identifican ambos conceptos desde un plano cognitivo. En concreto, este autor afirma que la satisfacción es el componente cognitivo de la felicidad: «La evidencia empírica que ha sido recogida, apunta hacia la existencia de una única medible dimensión de felicidad. Algunas medidas enfatizan su lado emocional - sentimientos de buen humorotras enfatizan el lado cognitivo, reflexivo -informes sobre satisfacción con la vida $\longrightarrow$ (p. 2).

Este aspecto relativo al carácter cognitivo de la satisfacción ha levantado cierta polémica, siendo cuestionado, como se verá más adelante, por diversos autores.

Desde la perspectiva de las Ciencias Sociales, quizá la inaprehensibilidad del concepto felicidad, que implica su dificultad de definición y operativización, haya contribuido a un decaimiento a la hora de estudiar este concepto dentro 
de la Psicología; aunque como afirman Kammann, Farry y Herbison (1984), quizá el surgimiento del paradigma conductista haya tenido que ver en este decaimiento, y haya recibido, sin embargo, mayor atención el estudio de la satisfacción; al ser probablemente este último de carácter más coyuntural que la felicidad. La discusión, por tanto, se centrará más en torno al concepto de satisfacción.

Ya Campbell, Converse y Rodgers (1976, p. 8) redogieron cómo el término satisfacción había sido ampliamente tratado desde perspectivas psicológicas y sociológicas: $« E l$ concepto de satisfacción ha estado sujeto a mucha investigación y teoría, tanto desde el tradicional "nivel de aspiración" de Kurt Lewin como desde la "privación relativa de Merton". El nivel de satisfacción puede ser definido precisamente como la discrepancia percibida entre aspiración y logro, yendo desde la percepción de realización hasta la de privación».

Esta definición lleva a Campbell y a sus colegas, en el trabajo citado, a concluir que «la satisfacción implica una experiencia de juicio o cognitiva; mientras que la felicidad sugiere una experiencia de sentimiento o afecto» (p. 8); opinión que es compartida por otros autores como Andrews y Withey (1976) y Andrews y McKennell (1980); que utilizan juicios de satisfacción y de felicidad como indicadores de bienestar, afirmando que los primeros son de carácter cognitivo mientras que los segundos son afectivos.

No obstante, hay algunas cuestiones que hacen pensar en una conclusión contraria a la señalada por estos autores, y que apunta, por tanto, en la dirección de considerar la satisfacción como un proceso afectivo más que cognitivo.

En sus comentarios a los resultados de «The American Soldier», Merton (1964) afirma que precisamente el concepto de «privación relativa» fue utilizado «para ayudar a explicar los sentimientos de insatisfacción, particularmente en casos en que no parecía probable, a primera vista, que la situación objetiva provocara tales sentimientos» (p. 239). Es decir, se está aludiendo al concepto satisfacción desde el punto de vista afectivo, de los sentimientos, y no como algo cognitivo.

Por otra parte, la satisfacción podría identificarse a la experiencia o sentimiento de éxito en la ejecución de un logro determinado, siguiendo la teoría del «nivel de aspiración» de Lewin (1978); siendo por tanto la satisfacción considerada desde esta perspectiva como un estado eminentemente positivo en el que se encuentra el individuo y, por tanto, de carácter afectivo. De hecho, en el establecimiento del nivel de aspiración, proceso que parece claramente cognitivo, está fuertemente implicado el miedo al fracaso, que será de mayor o menor intensidad en función de que el sujeto sitúe la tarea a realizar en un plano más real o menos, respectivamente (Frank, 1935).

Así pues, las conclusiones de los autores antes citados de que la satisfacción es una experiencia cognitiva son en alguna medida discutidas desde la hermenéutica realizada sobre los trabajos de Merton y Lewin. Según se acaba de discutir, parece que afecto y cognición se hayan íntimamente imbricados en mecanismos que llevan al sujeto a experimentar un sentimiento de éxito o fracaso.

Otra posición similar a la planteada por Campbell y sus colegas sería la defendida por Argyle (1987), como ya se comentó anteriormente. En su trabajo, el autor recoge algunas teorías sobre el concepto de satisfacción, siendo las que más aceptación han tenido aquellas que consideran la satisfacción como: a) el resultado de la ejecución o el logro de ciertas aspiraciones; definición similar a la considerada por autores como Levi y Andersson $(1980)$ y que estaría en la línea que exponen Campbell, Converse y Rodgers (1976); b) el estado que surge cuando comparamos nuestra vida con la de otros, es decir, basándose en las teo- 
rías de la comparación social, y c) según los postulados de la teoría de la adaptación, el grado de satisfacción con la vida de un individuo vendría dado en función de un nivel previo de adaptación a estímulos pasados. Según esta teoría, una misma condición objetiva puede resultar satisfactoria o no dependiendo de la adaptación a la misma en un momento anterior.

Lo que parece que queda implícito en todas estas teorías es que la satisfacción proviene de procesos evaluativo-cognitivos tales como la adaptación o la comparación social e intrapersonal (Smith, Diener y Wedell, 1989) y conductuales como la ejecución de ciertos logros. Lo que Michalos (1991) ampliaría en su «Teoría de las Discrepancias Múltiples» ${ }^{4}$. Así, cuando un individuo emite un juicio de satisfacción, es decir, responde a la pregunta de si se encuentra o no satisfecho, se ponen en marcha mecanismos cognitivos de evaluación, comparación, etc. Pero, según se comentó en párrafos anteriores, el estado en sí que supone la satisfacción parece claramente afectivo, o al menos de carácter emocional.

Hay autores que consideran la satisfacción desde un punto de vista afectivo (Weidemann y Anderson, 1985; en el caso de la satisfacción con el ambiente residencial) y otros que incluso no encuentran evidencia empírica con respecto a que los juicios de satisfacción sean cognitivos, pudiendo estar más determinados por el estado de humor del sujeto que responde (Schwarz y Clore, 1983).

La postura aquí defendida se orienta hacia la idea de que la satisfacción es, en definitiva, un sentimiento positivo, y por tanto un afecto. Cuando un individuo realiza un juicio de satisfacción está expresando verbalmente su actitud. De esta forma, el estudio de la satisfacción como indicador subjetivo de calidad de vida, debería abordarse desde una perspectiva actitudinal, considerando, por tanto, mecanismos cognitivos, afectivos y conductuales en el proceso por el cual un individuo manifiesta estar satisfecho o no con un aspecto concreto, o con su propia vida en general.

No obstante, y como se comentaba a comienzo de este epígrafe, el intento de separar los procesos cognitivos y afectivos puede resultar un tanto baldío. Como afirma Lazarus (1991) la concepción aristotélica en la que se basa la cultura occidental ha tendido siempre a separar cognición y emoción; el primero considerándolo como un proceso superior y el segundo como un proceso primitivo.

Esta tendencia a separar ambos sistemas proveniente de nuestra tradición cultural y de nuestras bases filosóficas, se ha visto claramente reflejada en el quehacer científico de forma que cognición y emoción se han estudiado como procesos separados, entendiéndose desde la perspectiva tradicional la emoción como una consecuencia de la cognición; o desde perspectivas más recientes en que la emoción puede ser entendida como un antecedente de la emoción ( $\mathrm{Za}$ jonc, 1980).

Parece, pues, que la cuestión no estriba en ver qué proceso tiene su ocurrencia en primer lugar, sino que ambos son procesos íntimamente interrelacionados, «elementos de un continuo, de un flujo inseparable de conducta» (Lewis, Sullivan y Michalson, 1984, p. 264) y, por tanto, así han de ser considerados. 


\section{Notas}

${ }^{1}$ Una visión del tema por distintos profesionales españoles fue recogida por Salustiano del Campo (1972) a través de una mesa redonda que dirigió bajo el patrocinio de la fundación FOESSA.,

${ }^{2}$ En general, existe una importante problemática de cara a encontrar instrumentos válidos de medida de la satisfacción y la felicidad. Detenerse a considerarla, sería extenderse demasiado dados los límites de este artículo. No obstante, el lector interesado puede encontrar una revisión reciente en Michalos (1991).

${ }^{3}$ Cfr. «Diccionario de Uso del Español». María Moliner.

" Según este autor la satisfacción y la felicidad son función de siete discrepacinas percibidas: «Lo que el individuo posee y lo que desea; lo que posee él y lo que posee el vecino; lo que tiene y lo que se merece; lo que tiene y lo que necesita; lo mejor que tuvo en el pasado; lo que espera tener en este momento y lo que esperará tener el futurom (Michalos, 1991, p. 5).

\section{Referencias}

Ajzen, I. (1988). Attitudes, Personality and Bebavior. Open University Press.

Andrews, F. M., y WITHeY, S. B. (1976). Social Indicators of well-being. American's percep. tions of life quality. Nueva York. Plenum Press.

Andrews, F. M., y Mckennelt, A. C. (1980). Measures of self-reported well-being: Their affective, cognitive and other components. Social Indicators Research, 8, 127-155.

Aranguren, J. L. L. (1989). Etica de la Felicidad y otros Lenguajes. Madrid. Tecnos.

ARGYLE, M. (1987). The Psychology of Happiness. Londres y Nueva York Methuen \& Co. LTD.

Blanco, A. (1985). La calidad de vida: Supuestos Psicosociales. En J. F. Morales; A. Blanco; C. Huici y J. M. Fernández (Comps.). Psicología Social Aplicada. Bilbao. Desclee De Brower.

Campbell, A.; Converse, P., y Rodgers, W. (1976). The Quality of American Life: Perceptions, evaluations and Satisfactions. Nueva York. Russell Sage Foundation.

Cantril, H. (1965). The Paitern of Human Concerns. Nueva Jersey. Rutgers University Press.

Del Campo, S. (1972). Los Indicadores Sociales a Debate. Madrid. (Fundación FOESSA). Euramérica.

Diaz, C. (1987). Eudaimonía. La felicidad como utopia necesaria. Madrid. Ediciones Encuentro.

Dirección General del Medio Ambiente (1979). Encuesta sabre la calidad de vida en España. Madrid. M.O.P.U. C.I.M.A.

FRANK, J. D. (1935). Some psychological determinants of the level of aspiration. The American Journal of Psychology, 47, 285-293.

Jiménez Burillo, F.; Aragonés, J. I.; Blanco, A.; Gil, F., y Torregrosa, J. R. (1984). La Psicología: Contribución al diseño de una sociedad con calidad de vida. I Congreso del Colegio Oficial de Psicólogos. Ponencias. pp. 87-106. Madrid. Colegio Oficial de Psicólogos.

Kammann, R.; Farry, M., y Herbison, P. (1984). The analysis and measurement of happiness as a sense of well-being. Social Indicators Research, 15, 91-115.

Lazarus, R. S. (1991). Cognition and motivation in emotion, American Psychologist, 46, 352-367.

Levi, L., y Andersson, L. (1980). La Tensión Psicosocial. Población, Ambiente y Calidad de la vida. México. El Manual Moderno.

Lewin, K. (1978). La Teoría del Campo en la Ciencia Social. Buenos Aires. Paidós.

Lewis, M.; Sullivan, M. W., y Mrchalson, L. (1984). The cognitive-emotional fugue. En C. E. Izard, J. Kagan y R. B. Zajonc (Eds.). Emotions, Cognitions \& Behavior. Cambridge. Cambridbe Univ. Press.

Merton, R. K. (1964). Teoría y Estructura Sociales. México. Fondo de Cultura Económica.

Michalos, A. C. (1991). Global Report on Student Well-Being. Life Satisfaction and Happiness. Nueva York. Springer-Verlag.

Morx, M. (1986). Bienestar Social. Madrid. Trivium.

Pena, J. B. (1977). Problemas de la medición del bienestar y conceptos afines (Una aplicación al caso español). Madrid. Presidencia del Gobierno. Instituto Nacional de Estadística.

SCHWARZ, N., y ClORE, G. L. (1983). Mood, misattribution, and judgments of well-being: Informative and directive functions of affective states. Joumal of Personality and Social Psychology, 45 (3), 513-523. 
Smith, R. H.; Diener, E., y Wedell, D. H. (1989). Intrapersonal and Social Comparison Determinants of Happiness: A Range-Frequency Analysis. Joumal of Personality and Social Psychology, 56, 317-325.

W ton. MTP Press Limited.

Weidemann, S., y Anderson, J. R. (1985). A conceptual framework for residential satisfaction. En: I. Altman y C. H. Werner (Eds.). Home Environments. Nueva York. Plenum Press.

Zajonc, R. B. (1980). Feeling and thinking: preferences need no inferences. American Psychologist, 35, 151-175. 